\title{
Article
}

\section{Effects of shear deformation and rotary inertia on the dynamics of anisotropic plates traversed by moving concentrated load}

\author{
Olorunsola Oriola Niyi ${ }^{1, *}$, Yomi Monday Aiyesimi ${ }^{2}$, Mohammed Jiya $^{3}$, Abdulhakeem Yusuf ${ }^{2}$ and \\ Gbolahan Bolarin ${ }^{2}$ \\ 1 Department of Mathematics, Federal College of Education, Kontagora, Nigeria. \\ 2 Department of Mathematics, Federal University of Technology Minna, Nigeria.; \\ yomi_aiyesimi2007@yahoo.com(Y.M.A); yusuf.abdulhakeem@futminna.edu.ng(A.Y); \\ g.bolarin@futminna.edu.ng(G.B) \\ 3 Department of Mathematics, University of Gambia, Sere Kunda, Gambia.; mjiya@utg.edu.gm \\ * Correspondence: s.niyi@fcekg.edu.ng
}

Received: 13 May 2019; Accepted: 30 August 2019; Published: 19 October 2019.

\begin{abstract}
The effects of shear deformation and rotary inertia on the dynamics of anisotropic plates traversed by varying moving load resting on Vlasov foundation is investigated in this work. The problem is solved for concentrated loads with simply supported boundary conditions. An analytic solution based on the Galerkin's method is used to reduce the fourth order partial differential equation into a system of coupled fourth order differential equation and a modification of the Struble's technique and Laplace transforms are used to solve the resulting fourth order differential equation. Results obtained indicate that shear deformation and rotary inertia have significant effect on the dynamics of the anisotropic plate on the Vlasov foundation. Solutions are obtained for both the moving force and the moving mass problems. From the graphical results obtained, the amplitude of vibrations of the plate under moving mass is greater than that of the moving force and increasing the value of rotary inertia $R_{0}$ reduces the amplitude of vibration of the plate. increasing the mass ratio increases the amplitude of vibration of the plate.
\end{abstract}

Keywords: Anisotropic plate, shear deformation, rotary inertia, Vlasov foundation, moving force, moving mass.

\section{Introduction}

$\mathbf{P}^{\text {his }}$ lates are widely used structures with wide engineering applications in aircrafts, nuclear vessels, hydraulics, bridges and roads. There has been a great deal of research on the analysis of structures (shells, plates and beams) with consideration for various factors such as displacements, thickness variation, stresses, curvature, effect of surrounding media, loads and masses. In particular, the problem of moving masses and loads over plates and beams has been a subject of investigation in Mathematics, Physics and Engineering because of its extensive use in everyday life.

Several authors have in particular investigated the effect of shear deformations and rotary inertia on plates and beams including $[1,2]$ with focus on isotropic plates. There has been very little focus on anisotropic plates and in particular the effect of shear deformations and rotary inertia, with varying masses transversing the plates. According to Toorani and Lakis [3], transverse shear deformation plays a very important role in reducing the effective flexural stiffness of anisotropic laminated plates and shells because their in-plane elastic modulus to transverse shear modulus ratio is high. Toorani et al. [3] based on Kirchhoff-Love assumptions opined that shear deformation is more significant in laminated anisotropic plates than isotropic constructions. The researchers, in $[1,2,4]$ worked on the effect of shear deformation and rotary inertia on anisotropic plates with consideration for flexural vibrations, wave amplitude and natural frequencies but not on moving loads. The problem moving loads transversing plates have received little attention unlike the effect of moving loads on isotropic plates and beams have also been studied by authors including [5-7] have given solutions using analytic and approximate methods such as he finite difference, Galerkin, Rayleigh-Ritz, transfer matrix and finite element methods. Kocaturk [8] studied rectangular anisotropic (orthotropic) plates on a tensionless 
elastic foundation and Ozgan [9] modeled laminated orthotropic plate-foundation interaction subjected to moving load using Vlasov model.

The present study modifies the Mindlin plate model which also incorporates both shear deformation and rotary inertia. The study employs varying flexural rigidity and varying mass per unit area in order to make the new model anisotropic.

This study is concerned with the effects of shear deformation and rotary inertia on the dynamics of moving concentrated masses on anisotropic plate resting on Vlasov foundation with simply supported boundary conditions.

\section{Problem formulation}

The general single equation of a plate which considered the influence of rotary inertia and shear on flexural motions of elastic plates was given by [4]. The elastic Vlasov foundation $Q(\xi, \eta, \tau)$ from $[10,11]$ is given by:

$$
Q(\xi, \eta, \tau)=\left(k-G_{d} \nabla^{2}\right) U(\xi, \eta, \tau),
$$

where $k$ and $G$ depict the foundation modulus and shear deformation parameter of the elastic Vlasov foundation. Anisotropy of the plate: two mechanical properties of the plate are varying in different directions on the rectangular plate. The flexural rigidity of the plate $D_{d}$ given by;

$$
D_{d}(\xi, \eta)=D_{0}\left(1-\frac{2 \xi}{a}+\frac{2 \xi^{2}}{a^{2}}\right)\left(1-\frac{2 \eta}{b}+\frac{2 \eta^{2}}{b^{2}}\right)
$$

and the mass per unit area of the plate $\mu_{d}$ given by;

$$
\mu_{d}(\xi, \eta)=\mu_{0}\left(1-\frac{2 \xi}{a}+\frac{2 \xi^{2}}{a^{2}}\right)\left(1-\frac{2 \eta}{b}+\frac{2 \eta^{2}}{b^{2}}\right) .
$$

\subsection{Governing equation}

The equation governing the anisotropic plate is given as;

$$
\begin{aligned}
& \left.\left.\left(D_{d}(\xi, \eta) \nabla^{2}-\left(\frac{\mu_{d}(\xi, \eta) D_{d}(\xi, \eta)}{h G_{d}}+R_{0}\right) \frac{\partial^{2}}{\partial \tau^{2}}\right) \nabla^{2}\right) U(\xi, \eta, \tau)+\frac{\mu_{d}(\xi, \eta) R_{0}}{h G_{d}}\right) \frac{\partial^{4} U(\xi, \eta, \tau)}{\partial \tau^{4}} \\
& +\mu_{d}(\xi, \eta) \frac{\partial^{2} U(\xi, \eta, \tau)}{\partial \tau^{2}}+\left(k-G_{f} \nabla^{2}\right) U(\xi, \eta, \tau) \\
& =M g \delta\left(x-v_{\xi} t\right) \delta\left(y-v_{\eta} t\right)\left(\frac{1}{g}\left[\frac{\partial^{2}}{\partial \tau^{2}}+\left(2 V \frac{\partial}{\partial \tau}+a\right)\left(\frac{\partial}{\partial x}+\frac{\partial}{\partial y}\right)+V^{2} \nabla^{2}\right] U(\xi, \eta, \tau)\right),
\end{aligned}
$$

where $U(\xi, \eta, \tau)$ is the displacement of the plate $\xi$ and $\eta$ are spatial coordinates, $\tau$ is the time coordinate, $D_{d}$ is the variable flexural rigidity of the plate, $\mu_{d}$ is the variable mass per unit area of the plate and $D_{0}$ is the constant flexural rigidity of the plate, $\mu_{0}$ is the constant mass per unit area of the plate. $R_{0}$ is the rotary inertia correction factor, $G_{d}$ is the shear modulus, $P(\xi, \eta, \tau)$ is the load, $M$ is the mass of the load $v_{\xi}$ and $v_{\eta}$ are the velocity components of the load.

\subsection{Dimensionless form}

The following dimensionless variables are introduced;

$$
x=\frac{\xi}{a}, \quad y=\frac{\eta}{b}, \quad t=\frac{\tau}{t_{0}}
$$

where $t_{0}$ will be specified and

$$
\mu=\frac{\mu_{d}}{\rho h^{\prime}} \quad D=\frac{D_{d}}{E h^{3}}, \quad \kappa=\frac{k h^{3}}{D}, \quad P_{0}=\frac{M g h^{2}}{D}, \quad G=\frac{G_{d} h^{3}}{D}, \quad V_{0}=\frac{V \tau}{h}, \quad a_{0}=\frac{a \tau^{2}}{h} .
$$

Substituting Equations (5) and (6) into Equation (4) and making some rearrangements yields: 


$$
\begin{aligned}
& D_{1}\left(F_{x}+F_{y}\right)\left(\frac{\partial^{2} U(x, y, t)}{\partial x^{2}}+\frac{\partial^{2} U(x, y, t)}{\partial y^{2}}\right)-b_{1}\left(F_{x} F_{y}\right)^{2}\left(\frac{\partial^{4} U(x, y, t)}{\partial t^{2} \partial x^{2}}+\frac{\partial^{4} U(x, y, t)}{\partial t^{2} \partial y^{2}}\right) \\
& -R_{0}\left(\frac{\partial^{4} U(x, y, t)}{\partial t^{2} \partial x^{2}}+\frac{\partial^{4} U(x, y, t)}{\partial t^{2} \partial y^{2}}\right)+b_{2} \mu(x, y) \frac{\partial^{4} U(x, y, t)}{\partial t^{4}}+b_{3} \mu_{0} F_{x} F_{y} \frac{\partial^{2} U(x, y, t)}{\partial t^{2}}+\kappa_{f} U(x, y, t) \\
& -G_{f}\left(\frac{\partial^{4} U(x, y, t)}{\partial t^{2} \partial x^{2}}+\frac{\partial^{4} U(x, y, t)}{\partial t^{2} \partial y^{2}}\right)=P_{0} \delta\left(x-v_{\xi} t\right) \delta\left(y-v_{\eta} t\right)-\frac{P_{0}}{g} \delta\left(x-v_{\xi} t\right) \delta\left(y-v_{\eta} t\right) \\
& {\left[b_{3} \frac{\partial^{2} U(x, y, t)}{\partial t^{2}}+\left(c_{0} \frac{\partial}{\partial t}+a_{0}\right)\left(\frac{\partial}{\partial x}+\frac{\partial}{\partial y}\right) U(x, y, t)+V_{0}^{2}\left(\frac{\partial^{2} U(x, y, t)}{\partial x^{2}}+\frac{\partial^{2} U(x, y, t)}{\partial y^{2}}\right)\right],}
\end{aligned}
$$

where

$$
\left.\begin{array}{rl}
D_{1} & =\frac{4 D_{0}}{E h^{5}}, b_{1}=\frac{\mu_{0} D_{0}}{E G D \rho^{2} t_{0}^{2}}, b_{2}=\frac{R_{0} h^{6}}{G D t_{0}^{4}}, b_{3}=\frac{h^{6}}{t_{0}^{2}}, k_{f}=\frac{\kappa D}{h^{2}}, G_{f}=\frac{G D}{h^{3}}, V_{0}=\frac{V h^{2}}{t_{0}^{2}}, a_{0}=\frac{a h^{2}}{t_{0}^{2}}, \\
c_{0} & =\frac{2 V_{0}}{t_{0}}, F_{x}=1-2 x+2 x^{2} F_{y}=1-2 y+2 y^{2} F_{x y}^{2}=\left(1-2 x+2 x^{2}\right)^{2}\left(1-2 y+2 y^{2}\right)^{2} .
\end{array}\right\}
$$

Also the varying flexural rigidity and varying mass per unit area becomes:

$$
\left.\begin{array}{l}
D(x, y)=\frac{D_{0}}{h^{3}}\left(1-2 x+2 x^{2}\right)\left(1-2 y+2 y^{2}\right) \\
\mu(x, y)=\frac{\mu_{0}}{\rho h}\left(1-2 x+2 x^{2}\right)\left(1-2 y+2 y^{2}\right)
\end{array}\right\}
$$

Considering the case (simply supported) with the boundary conditions:

$$
\left.\begin{array}{c}
U(0, y, t)=0=U(1, y, t)=0 ; \quad U_{x x}(0, y, t)=0=U_{x x}(1, y, t)=0 ; \text { for } 0 \leq x \leq 1 \\
\text { and } U(x, 0, t)=0=U(x, 1, t)=0 ; \quad U_{y y}(x, 0, t)=0=U_{y y}(x, 1, t)=0 ; \text { for } 0 \leq y \leq 1 .
\end{array}\right\}
$$

The initial condition taken as;

$$
\left.\begin{array}{rr}
\left.U(x, y, t)\right|_{t=0}=U_{0},\left.\quad U_{t}(x, y, t)\right|_{t=0}=0 \\
\left.U_{t t}(x, y, t)\right|_{t=0}=0,\left.\quad U_{t t t}(x, y, t)\right|_{t=0}=0
\end{array}\right\}
$$

\section{Method of Solution}

The method of analysis involved in solving (7) subject to conditions (10) and (11) requires the use of the Galerkin's method to separate variables and reduce the fourth order partial differential equation governing plates to a set of coupled fourth order ordinary differential equation. Also the Dirac-Delta function is expressed as a Fourier series Cosine. In order to solve (7), the displacement written in the form:

$$
U(x, y, t)=\sum_{m=1}^{\infty} \Lambda_{m}(x, y) y_{m}(t)
$$

where $\Lambda_{m}(x, y)$ are the known eigen-functions of the plate with the same boundary conditions. We obtain the value of $\Lambda_{m}(x, y)$ by considering the free vibration of rectangular plates given by;

$$
\nabla^{4} \Lambda_{m}-\frac{\mu}{D} \Omega_{m}^{2} \Lambda_{m}=0
$$

where

$$
\frac{\mu}{D} \Omega_{m}^{2} \Lambda_{m}=W_{m}^{4}
$$

$\Omega_{m}, m=1,2,3 \ldots$ are the natural frequencies of the dynamic system and $y_{m}(t)$ are amplitude functions which have to be solved. $\Lambda_{n}(x, y)$ are assumed to be products of the function $\phi_{n i}(x)$ and $\phi_{n j}(y)$ which are plate functions in the direction of axes respectively. Hence

$$
\Lambda_{n}(x, y)=\phi_{n i}(x) \phi_{n j}(y)
$$


Each of these plate functions satisfies all the boundary conditions in its direction respectively. In particular, these plate functions can be defined respectively as:

$$
\left.\begin{array}{l}
\phi_{n i}(x)=\sin \frac{\psi_{n i} x}{L_{x}}+A_{n i} \cos \frac{\psi_{n i} x}{L_{x}}+B_{n i} \sinh \frac{\psi_{n i} x}{L_{x}+}+C_{n i} \cosh \frac{\psi_{n i x} x}{L_{x}} ; \\
\phi_{n j}(y)=\sin \frac{\psi_{n i} y}{L_{y}}+A_{n j} \cos \frac{\psi_{n i y}}{L_{y}}+B_{n j} \sinh \frac{\psi_{n j} y}{L_{y}+}+C_{n j} \cosh \frac{\psi_{n j} y}{L_{y}},
\end{array}\right\}
$$

where $A_{n i}, B_{n i}, C_{n i}, A_{n j}$ and $B_{n j}, C_{n j}$ are constants determined by the boundary conditions. $\psi_{n i}$ and $\psi_{n j}$ are called modal frequencies. When the anisotropic plate has arbitrary end support conditions since the plate under consideration has simple support at all its edges, (16) becomes:

$$
\phi_{n i}(x)=\sin \frac{n_{i} \pi x}{L_{x}}, \quad \phi_{n j}(y)=\sin \frac{n_{j} \pi y}{L_{y}} .
$$

Therefore the non-dimensional plate function is given as:

$$
\phi_{n i}(x)=\sin n_{i} \pi x, \quad \phi_{n j}(y)=\sin n_{j} \pi y .
$$

\subsection{Transformation of the governing equation}

By applying the Generalized Galerkin's method Equation (7) is transformed to

$$
\begin{aligned}
& \sum_{m=1}^{\infty}\left[\left(\frac{D_{1}}{\mu_{0}}\left(F_{x}+F_{y}\right)\right)\left[\Lambda_{m, x x}(x, y) y_{m}(t)+\Lambda_{m, y y}(x, y) y_{m}(t)\right]\right. \\
& -\frac{b_{1}\left(F_{x} F_{y}\right)^{2}}{\mu_{0}}\left[\Lambda_{m, x x}(x, y) \ddot{y}_{m}(t)+\Lambda_{m, y y}(x, y) \ddot{y}_{m}(t)\right]-\frac{R_{0}}{\mu_{0}}\left[\Lambda_{m, x x}(x, y) \ddot{y}_{m}(t)+\Lambda_{m, y y}(x, y) \ddot{y}_{m}(t)\right] \\
& +\frac{b_{2}}{\mu_{0}} \mu(x, y) \Lambda_{m}(x, y) \dddot{y}_{m}(t)+b_{3} F_{x} F_{y} \Lambda_{m}(x, y) \ddot{y}_{m}(t)+\frac{k_{f}}{\mu_{0}} \Lambda_{m}(x, y) y_{m}(t) \\
& -\frac{G_{f}}{\mu_{0}}\left[\Lambda_{m, x x}(x, y) y_{m}(t)+\Lambda_{m, y y}(x, y) y_{m}(t)\right]+\frac{P_{0}}{\mu_{0} g} \delta\left(x-v_{\xi} t\right) \delta\left(y-v_{\eta} t\right)\left(b_{3} \Lambda_{m}(x, y) \ddot{y}_{m}(t)\right) \\
& \left(b_{3} \Lambda_{m}(x, y) \ddot{y}_{m}(t)\right)+c_{0}\left[\Lambda_{m, x}(x, y) \dot{y}_{m}(t)+\Lambda_{m, y}(x, y) \dot{y}_{m}(t)\right]+a_{0}\left[\Lambda_{m, x}(x, y) y_{m}(t)+\Lambda_{m, y}(x, y) y_{m}(t)\right] \\
& \left.+V_{0}^{2}\left[\Lambda_{m, x x}(x, y) y_{m}(t)+\Lambda_{m, y y}(x, y) y_{m}(t)\right]=\frac{P_{0}}{\mu_{0} g} \delta\left(x-v_{x} t\right) \delta\left(y-v_{y} t\right)\right]
\end{aligned}
$$

where

$$
\left.\begin{array}{c}
\Lambda_{m, x}(x, y)=\frac{\partial}{\partial x} \Lambda_{m}(x, y), \quad \Lambda_{m, y}(x, y)=\frac{\partial}{\partial y} \Lambda_{m}(x, y) \\
\Lambda_{m, x x}(x, y)=\frac{\partial^{2}}{\partial x^{2}} \Lambda_{m}(x, y), \quad \Lambda_{m, y y}(x, y)=\frac{\partial^{2}}{\partial y^{2}} \Lambda_{m}(x, y) \\
\dot{y}_{m}(t)=\frac{d y_{m}(t)}{d t}, \quad \ddot{y}_{m}(t)=\frac{d^{2} y_{m}(t)}{d t^{2}}, \quad \dddot{y}_{m}(t)=\frac{d^{4} y_{m}(t)}{d t^{4}} .
\end{array}\right\}
$$

Using the property of the Dirac-delta functions and expressing it in the Fourier Cosine series (because it is an even function) as,

$$
\begin{aligned}
& \delta\left(x-v_{x} t\right)=\frac{1}{L_{x}}\left(1+2 \sum_{j=1}^{\infty} \cos \frac{j \pi v_{x} t}{L_{x}} \cos \frac{j \pi x}{L_{x}}\right) \\
& \delta\left(y-v_{y} t\right)=\frac{1}{L_{y}}\left(1+2 \sum_{k=1}^{\infty} \cos \frac{k \pi v_{y} t}{L_{y}} \cos \frac{k \pi y}{L_{y}}\right) .
\end{aligned}
$$

Multiplying both sides of Equation (19) by $\Lambda_{n}(x, y)$ in view of the orthogonality of $\Lambda_{m}(x, y)$ and integrating on Area (A) of the plate yields, the simplified equation: 


$$
\begin{aligned}
& \frac{d^{4} y_{p}(t)}{d t^{4}}+\alpha_{1}^{2} \frac{d^{2} y_{p}(t)}{d t^{2}}+\alpha_{2}^{2} y_{p}(t)+\epsilon_{0}\left[\left(I_{01} I_{02}+2 \sum_{j=1}^{\infty} \cos j \pi v_{x} t I_{41}^{j} I_{02}+2 \sum_{k=1}^{\infty} \cos k \pi v_{y} t I_{42}^{k} I_{01}\right.\right. \\
& \left.+4 \sum_{j=1}^{\infty} \sum_{k=1}^{\infty} \cos j \pi v_{x} t \cos k \pi v_{y} t I_{41}^{j} I_{42}\right) \frac{d^{2} y_{p}(t)}{d t^{2}}+\left(a_{1} I_{51} I_{02}+b_{1} I_{01} I_{52}+2 \sum_{j=1}^{\infty} \cos j \pi v_{x} t\left(a_{1} I_{51}^{j} I_{02}+b_{1} I_{41}^{j} I_{52}\right)\right. \\
& \left.+2 \sum_{k=1}^{\infty} \cos k \pi v_{y} t\left(a_{1} I_{51} I_{42}^{k}+b_{1} I_{01} I_{52}^{k}\right)+4 \sum_{j=1}^{\infty} \sum_{k=1}^{\infty} \cos j \pi v_{x} t \cos k \pi v_{y} t\left(a_{1} I_{51}^{j} I_{42}^{k}+b_{1} I_{41}^{j} I_{52}^{k}\right)\right) \\
& \times\left(c_{0} \frac{d y_{p}(t)}{d t}+a_{0} y_{p}(t)\right)+v_{0}\left(\left(a_{1}^{2}+b_{1}^{2}\right) I_{01} I_{02}+2 \sum_{j=1}^{\infty}\left(a_{1}^{2}+b_{1}^{2}\right) \cos j \pi v_{x} t I_{41}^{j} I_{02}\right. \\
& \left.\left.+2 \sum_{k=1}^{\infty}\left(a_{1}^{2}+b_{1}^{2}\right) \cos k \pi v_{y} t I_{42}^{k} I_{01}+4 \sum_{j=1}^{\infty} \sum_{k=1}^{\infty}\left(a_{1}^{2}+b_{1}^{2}\right) \cos j \pi v_{x} t \cos k \pi v_{y} t I_{41}^{j} I_{42}^{k}\right) y_{p}(t)\right] \\
& =\frac{P_{0}}{\mu_{0} D_{3} I_{B 11} I_{B 12}} \sin n_{i} \pi v_{x} t \sin n_{j} \pi v_{y} t
\end{aligned}
$$

where

$$
\begin{gathered}
\epsilon_{0}=\frac{P_{0}}{L_{x} L_{y} \mu_{0} g}, \quad \alpha_{1}^{2}=\frac{B_{2}}{B_{1}}, \quad \alpha_{2}^{4}=\frac{B_{3}}{B_{1}} \\
B_{1}=D_{3} \int_{0}^{1} \int_{0}^{1} F_{x y} \sin p_{i} \pi v_{x} t \sin n_{i} \pi v_{x} t \sin p_{j} \pi v_{y} t \sin n_{j} \pi v_{y} t d y d x \\
B_{2}=D_{4} I_{B 11} I_{B 12}+D_{5}\left(a_{1}^{2}+b_{1}^{2}\right) I_{2 B 1} I_{2 B 2}+D_{6}\left(a_{1}^{2}+b_{1}^{2}\right) I_{01} I_{02} \\
B_{3}=-D_{7 a}\left(a_{1}^{2}+b_{1}^{2}\right) I_{B 11} I_{02}-D_{7 a}\left(a_{1}^{2}+b_{1}^{2}\right) I_{01} I_{12}+D_{k} I_{01} I_{02}-D_{G}\left(a_{1}^{2}+b_{1}^{2}\right) I_{01} I_{02} \\
D_{3}=\frac{b_{2}}{\mu_{0}}, \\
D_{4}=b_{3}, \\
D_{5}=\frac{b_{1}}{\mu_{0}}, \\
D_{6}=\frac{R_{0}}{\mu_{0}}, \\
D_{7}=\frac{D_{1}}{\mu_{0}}, \\
D_{k}=\frac{k_{f}}{\mu_{0}}, \\
D_{G}=\frac{G_{f}}{\mu_{0}} \\
I_{01}=\int_{0}^{1} \sin p_{i} \pi x \sin n_{i} \pi x d x \\
I_{02}=\int_{0}^{1} \sin p_{j} \pi y \sin n_{j} \pi y d y \\
I_{41}^{j}=\int_{0}^{1} \sin p_{i} \pi x \sin n_{i} \pi x \cos j \pi x d x, \\
I_{42}^{k}=\int_{0}^{1} \sin p_{j} \pi y \sin n_{j} \pi y \cos k \pi y d y \\
I_{51}=\int_{0}^{1} \cos p_{i} \pi x \sin n_{i} \pi x d x, \\
I_{52}=\int_{0}^{1} \cos p_{j} \pi y \sin n_{j} \pi y d y \\
I_{51}^{j}=\int_{0}^{1} \cos p_{i} \pi x \sin n_{i} \pi x \cos j \pi x d x, \\
I_{52}^{k}=\int_{0}^{1} \cos p_{j} \pi y \sin n_{j} \pi y \cos k \pi y d y \\
I_{B 11}=\int_{0}^{1}\left(1-2 x+2 x^{2}\right) \sin p_{i} \pi x \sin n_{i} \pi x d x, \\
I_{B 12}=\int_{0}^{1}\left(1-2 y+2 y^{2}\right) \sin p_{j} \pi y \sin n_{j} \pi y d y .
\end{gathered}
$$

Implementing the integrals above and substituting into Equation (23) yields;

$$
\frac{d^{4} y_{p}(t)}{d t^{4}}+\left(\alpha_{1}^{2}+\frac{\epsilon_{0}}{4}\right) \frac{d^{2} y_{p}(t)}{d t^{2}}+c_{0} \epsilon_{0} \Delta_{1}^{2} \frac{d y_{p}(t)}{d t}+\left(\alpha_{2}^{2}+\epsilon_{0} \Delta_{2}^{2}\right) y_{p}(t)=P_{f} \sin n_{i} \pi x \sin n_{j} \pi y,
$$

where

$$
\begin{aligned}
& \Delta_{1}^{2}=\frac{a_{1}\left[\left(p_{i}+n_{i}\right)(-1)^{p_{i}-n_{i}}-\left(p_{i}-n_{i}\right)(-1)^{p_{i}+n_{i}}-2 n_{i}\right]}{4 \pi\left(p_{i}^{2}-n_{i}^{2}\right)}+\frac{b_{1}\left[\left(p_{i}+n_{i}\right)(-1)^{p_{i}-n_{i}}-\left(p_{i}-n_{i}\right)(-1)^{\left.p_{i}+n_{i}-2 n_{i}\right]}\right.}{4 \pi\left(p_{i}^{2}-n_{i}^{2}\right)} \\
& +\frac{4 n_{i}}{\pi} \sum_{j=0}^{\infty} \cos j \pi v_{x} t\left(a_{1} \frac{n_{i}^{2}-p_{i}^{2}-j_{i}^{2}}{\left[j_{i}^{2}-\left(p_{i}+n_{i}\right)^{2}\right]\left[j_{i}^{2}-\left(p_{i}-n_{i}\right)^{2}\right]}\right)+\frac{4 n_{j}}{\pi} \sum_{k=0}^{\infty} \cos k \pi v_{y} t\left(a_{1} \frac{n_{j}^{2}-p_{j}^{2}-k_{j}^{2}}{\left[k_{j}^{2}-\left(p_{j}+n_{j}\right)^{2}\right]\left[k_{j}^{2}-\left(p_{j}-n_{j}\right)^{2}\right]}\right) \\
& \begin{array}{l}
\Delta_{2}^{2}=a_{1} \Delta_{1}^{2}-\frac{V_{0}\left(a_{2}^{2}+b_{2}^{2}\right)}{P_{0}} \\
\quad P_{f}=\frac{P_{0}}{\mu_{0} D_{3} I_{B 11} I_{B 12}} .
\end{array}
\end{aligned}
$$


Equation (25) is the transformed forth order differential equation which represents the problem of anisotropic plate on Vlasov foundation with simply supported boundary conditions. We consider two possible solution cases.

\subsection{Case I: Moving force}

An approximate model of the differential equation describing the response of anisotropic plates on Vlasov foundation is obtained by neglecting the inertia term $\epsilon_{0}=0$. Hence Equation (25) becomes:

$$
\frac{d^{4} y_{p}(t)}{d t^{4}}+\alpha_{1}^{2} \frac{d^{2} y_{p}(t)}{d t^{2}}+\alpha_{2}^{2} y_{p}(t)=P_{f} \sin n_{i} \pi x \sin n_{j} \pi y
$$

Subjecting (27) to a Laplace transform we us initial boundary conditions (11) yields;

$$
\begin{aligned}
& U(x, y, t)=\sum_{n_{i}=1}^{\infty} \sum_{n_{j}=1}^{\infty} \frac{1}{w_{f 1}^{2}-w_{f 2}^{2}}\left(\frac { P _ { f } } { 2 } \left(\frac { 1 } { ( w _ { f 2 } ^ { 2 } - \theta _ { 1 } ^ { 2 } ) ( w _ { f 2 } ^ { 2 } - \theta _ { 2 } ^ { 2 } ) } \left[\left(w_{f 2}^{2}-\theta_{2}^{2}\right)\left(\cos \theta_{1} t-\cos w_{f 2} t\right)\right.\right.\right. \\
& \left.-\left(w_{f 2}^{2}-\theta_{1}^{2}\right)\left(\cos \theta_{2} t-\cos w_{f 2} t\right)\right]\left(\frac { 1 } { ( w _ { f 1 } ^ { 2 } - \theta _ { 1 } ^ { 2 } ) ( w _ { f 1 } ^ { 2 } - \theta _ { 2 } ^ { 2 } ) } \left[\left(w_{f 1}^{2}-\theta_{2}^{2}\right)\left(\cos \theta_{1} t-\cos w_{f 1} t\right)\right.\right. \\
& \left.\left.\left.\left.\left.-\left(w_{f 1}^{2}-\theta_{1}^{2}\right)\left(\cos \theta_{2} t-\cos w_{f 1} t\right)\right]\right)+\left[\left(\alpha_{1}^{2}-w_{f 2}^{2}\right) \cos w_{f 2} t\right)-\left(\alpha_{1}^{2}-w_{f 1}^{2}\right) \cos w_{f 1} t\right)\right] y_{0}\right) \sin \frac{n_{i} \pi x}{l_{x}} \sin \frac{n_{j} \pi y}{l_{y}},
\end{aligned}
$$

which is the transverse displacement response to a moving varying force by a simply supported anisotropic plate on a Vlasov foundation.

$$
\left.\begin{array}{l}
w_{f 1}^{2}=\frac{1}{2}\left[\alpha_{1}^{2}-\sqrt{\alpha_{1}^{4}-4 \alpha_{2}^{2}}\right] \\
w_{f 2}^{2}=\frac{1}{2}\left[\alpha_{1}^{2}+\sqrt{\alpha_{1}^{4}-4 \alpha_{2}^{2}}\right]
\end{array}\right\}
$$

\subsection{Case II: Moving mass}

We consider the moving mass problem where the inertia term $\epsilon_{0}$ is not neglected. And Equation (25) remains;

$$
\frac{d^{4} y_{p}(t)}{d t^{4}}+\left(\alpha_{1}^{2}+\frac{\epsilon_{0}}{4}\right) \frac{d^{2} y_{p}(t)}{d t^{2}}+c_{0} \epsilon_{0} \Delta_{1}^{2} \frac{d y_{p}(t)}{d t}+\left(\alpha_{2}^{2}+\epsilon_{0} \Delta_{2}^{2}\right) y_{p}(t)=P_{f} \sin n_{i} \pi x \sin n_{j} \pi y .
$$

To solve (25), we use an approximate analytic solution a modification of the asymptotic method due to Strubble's technique as used by [12-14]. To this end, a modified frequency corresponding to the frequency of the free system due to the presence of the effect of the moving mass is sought by using:

$$
\frac{d^{2} y_{p}(t)}{d t^{2}}+\alpha_{m n}^{2} y_{p}(t)=0
$$

where

$$
\alpha_{m n}=\alpha_{2}\left[1-\frac{1}{2 \alpha_{1}^{4}}\left(\alpha_{1}^{2}\left(\alpha_{1}^{2}-1\right)+\frac{\epsilon_{0}}{\alpha_{2}^{2}}\left(\alpha_{2}^{2}-\Delta_{i j} \alpha_{1}^{2}\right)\right)\right]
$$

$\alpha_{m n}$ is called the modified natural frequency representing the frequency of the system due to the effect of the foundation. Hence, Equation (30) can be rewritten as:

$$
\frac{d^{4} y_{p}(t)}{d t^{4}}+\frac{d^{2} y_{p}(t)}{d t^{2}}+\alpha_{m n}^{2} y_{p}(t)=P_{f} \sin n_{i} \pi x \sin n_{j} \pi y .
$$

Solving (33) using Laplace transforms method subject to initial boundary conditions (11) yields: 


$$
\begin{aligned}
& U(x, y, t)=\sum_{n_{i}=1}^{\infty} \sum_{n_{j}=1}^{\infty} \frac{1}{w_{m 1}^{2}-w_{m 2}^{2}}\left(\frac { P _ { f } } { 2 } \left(\frac { 1 } { ( w _ { m 2 } ^ { 2 } - \theta _ { 1 } ^ { 2 } ) ( w _ { m 2 } ^ { 2 } - \theta _ { 2 } ^ { 2 } ) } \left[\left(w_{m 2}^{2}-\theta_{2}^{2}\right)\left(\cos \theta_{1} t-\cos w_{m 2} t\right)\right.\right.\right. \\
& \left.-\left(w_{m 2}^{2}-\theta_{1}^{2}\right)\left(\cos \theta_{2} t-\cos w_{m 2} t\right)\right]-\left(\frac { 1 } { ( w _ { m 1 } ^ { 2 } - \theta _ { 1 } ^ { 2 } ) ( w _ { m 1 } ^ { 2 } - \theta _ { 2 } ^ { 2 } ) } \left[\left(w_{m 1}^{2}-\theta_{2}^{2}\right)\left(\cos \theta_{1} t-\cos w_{m 1} t\right)\right.\right. \\
& \left.\left.\left.\left.\left.-\left(w_{m 1}^{2}-\theta_{1}^{2}\right)\left(\cos \theta_{2} t-\cos w_{m 1} t\right)\right]\right)+\left[\left(1-w_{m 1}^{2}\right) \cos w_{m 1} t\right)-\left(1-w_{m 2}^{2}\right) \cos w_{m 2} t\right)\right] y_{0}\right) \sin \frac{n_{i} \pi x}{l_{x}} \sin \frac{n_{j} \pi y}{l_{y}}
\end{aligned}
$$

which is the transverse displacement response to a moving varying mass by a simply supported anisotropic plate on a Vlasov foundation. Where

$$
\left.\begin{array}{l}
w_{m 1}^{2}=\frac{1}{2}\left[\alpha_{1}^{2}-\sqrt{1-4 \alpha_{m n}^{2}}\right. \\
w_{m 2}^{2}=\frac{1}{2}\left[\alpha_{1}^{2}+\sqrt{1-4 \alpha_{m n}^{2}}\right]
\end{array}\right\}
$$

\subsection{Resonance}

We consider the resonance which takes place when the displacement of the vibrating structure becomes unbounded. In actual practice, when this happens the structure would collapse as the intensive vibrations causes cracks or permanent deformation in the vibrating system. Therefore, the conditions under which the solutions (28) and (34) grow without bound are investigated. The anisotropic plate traversed by moving force in Equation (28) will reach the state of resonance whenever:

$$
\left.\begin{array}{c}
w_{f 1}=\theta_{1} a n d w_{f 2}=\theta_{1} \\
w_{f 2}=\theta_{2} a n d w_{f 2}=\theta_{2}
\end{array}\right\}
$$

Similarly, Equation (34) shows that the same plate under moving concentrated mass will experience resonance effect whenever:

$$
\left.\begin{array}{c}
w_{m 1}=\theta_{1} a n d w_{m 2}=\theta_{1} \\
w_{m 1}=\theta_{2} a n d w_{m 2}=\theta_{2} .
\end{array}\right\}
$$

From Equation (35)

$$
\frac{1}{2}\left[\alpha_{1}^{2}-\sqrt{1-4 \alpha_{m n}^{2}}\right]-\frac{1}{2}\left[\alpha_{1}^{2}+\sqrt{1-4 \alpha_{m n}^{2}}\right]=0
$$

Hence

$$
\alpha_{m n}= \pm \frac{1}{2}
$$

Similarly from 29

$$
\frac{1}{2}\left[\alpha_{1}^{2}-\sqrt{\alpha_{1}^{4}-4 \alpha_{2}^{2}}\right]-\frac{1}{2}\left[\alpha_{1}^{2}+\sqrt{\alpha_{1}^{4}-4 \alpha_{2}^{2}}\right]=0
$$

Hence

$$
\alpha_{1}^{2}= \pm 2 \alpha_{2}
$$

Now, from Equation (32), we have $\alpha_{m n}=\alpha_{2}\left[1-\frac{1}{2 \alpha_{1}^{4}}\left(\alpha_{1}^{2}\left(\alpha_{1}^{2}-1\right)+\frac{\epsilon_{0}}{\alpha_{2}^{2}}\left(\alpha_{2}^{2}-\Delta_{i j} \alpha_{1}^{2}\right)\right)\right]$. And

$$
\alpha_{2}\left(\frac{\alpha_{2}^{3}+2 \alpha_{2}^{2}-\epsilon_{0}\left(\alpha_{2}-2 \Delta_{i j}\right)}{8 \alpha_{2}^{3}}\right)<1 .
$$


From equation above, it is evident that for the same natural frequency, the critical velocity for the system of the plate moving force is greater than that of the moving mass problem. Thus, for the same natural frequency of the anisotropic plate, resonance is reached earlier in the moving mass than in the moving force system.

\section{Discussion and Analysis}

A rectangular plate of breadth $L_{x}=0.456$ and length $L_{y}=0.946$ was used as sample to carry out numerical experiments on the dynamics of the anisotropic plate. The velocity of the plate was assumed to be $0.8 \mathrm{~m} / \mathrm{s}$ and the Young's modulus $E=1 \times 10^{9} \mathrm{~kg} / \mathrm{m}^{2}$.

A comparison of the displacement of structure-load force and structure-load mass system of anisotropic plate on Vlasov foundation is given in Figure 1 showing a larger displacement for the moving mass system. While Figure 2 and Figure 3 show the effects of Rotary inertia $\left(R_{0}\right)$ on the anisotropic plate for the moving force and moving mass problems respectively. In both cases, increasing Rotary inertia $\left(R_{0}\right)$ reduces the displacement.

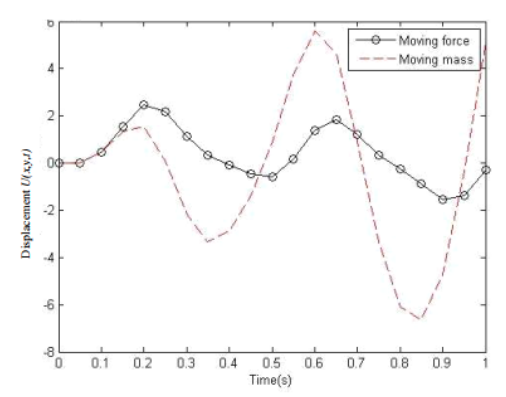

Figure 1. Moving Force vs Moving Mass

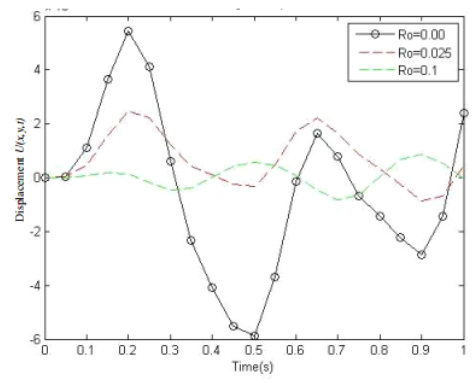

Figure 2. Effect of Rotary Inertia on Moving Mass

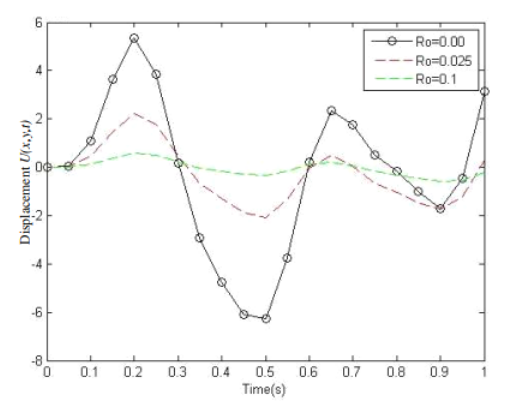

Figure 3. Effect of Rotary Inertia on Moving Force

Figure 4 shows the effect of mass ratio $\left(\epsilon_{0}\right)$ on the anisotropic plate for different values of $\left(\epsilon_{0}\right)$, increasing the mass ratio increases the displacement. 


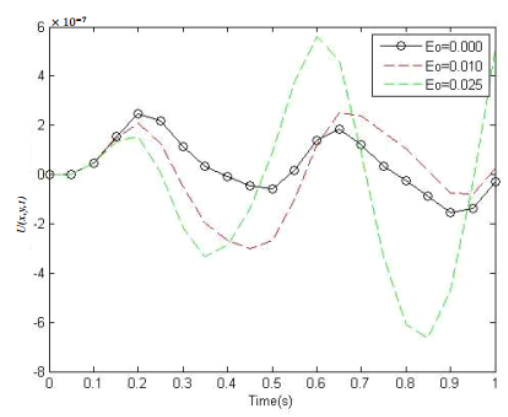

Figure 4. Effect of Mass Ratio

Figure 5, Figure 6 and Figure 7 are surface plots of the effect of shear modulus $G$ on the anisotropic plate for $G=0, G=2000$ and $G=6000$ respectively. Where $R_{0}=2.4, k=10, G_{f}=7.7$

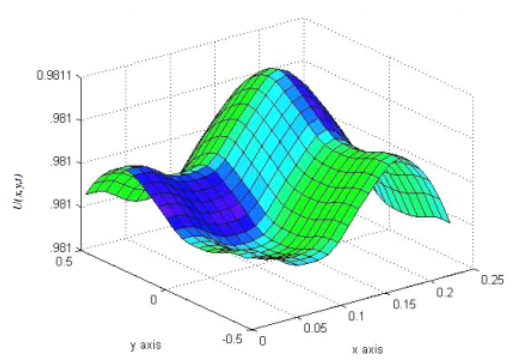

Figure 5. Shear $\operatorname{Modulus}(G)=0$

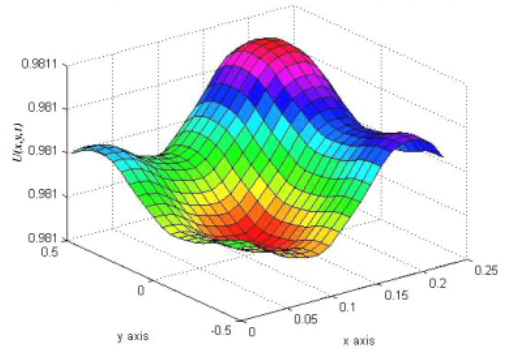

Figure 6. Shear Modulus $(G)=2000$

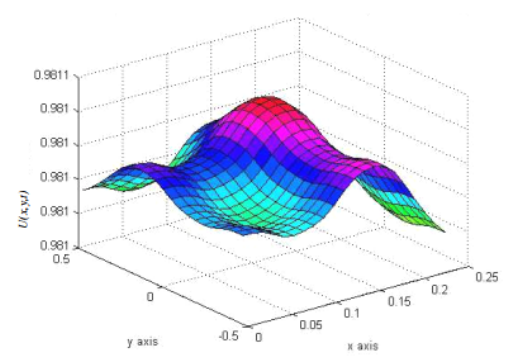

Figure 7. Shear Modulus $(G)=6000$

The effect of rotary inertia $\left(R_{0}\right)$ on the dynamics of the anisotropic plate on Vlasov foundation are shown for different values in Figure 8 Figure 9 and Figure 10 when the mass ratio $\left(\epsilon_{0}=0.5\right)$ 


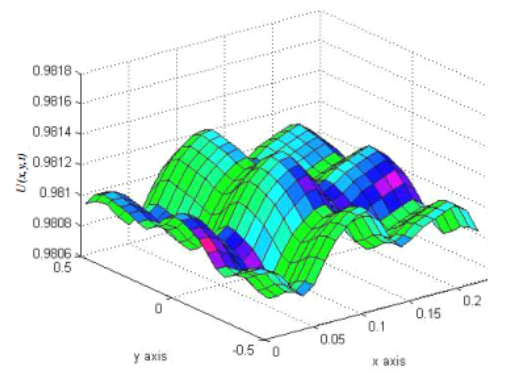

Figure 8. Rotary Inertia $\left(R_{0}\right)=0.01$

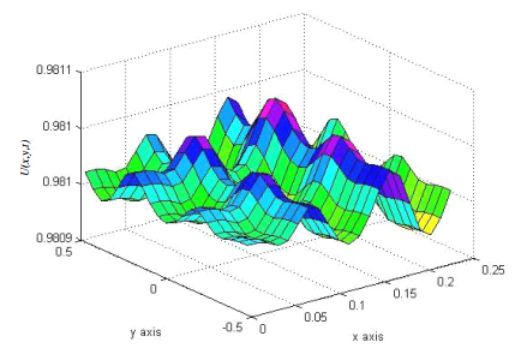

Figure 9. Rotary $\operatorname{Inertia}\left(R_{0}\right)=0.015$

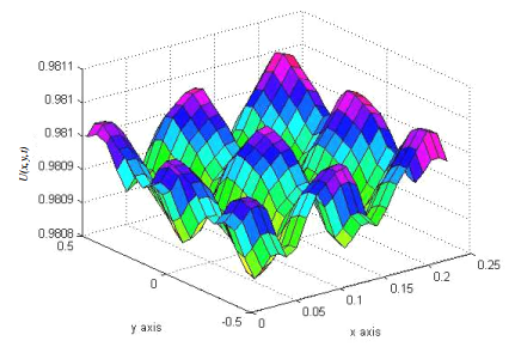

Figure 10. Rotary $\operatorname{Inertia}\left(R_{0}\right)=0.025$

Figure 11, Figure 12 and Figure 13 are the surface plots showing the effect of three different mass ratio $\left(\epsilon_{0}\right)$ on the dynamics of the anisotropic plate on Vlasov foundation where $R_{0}=2.4$, frequency $=200 \mathrm{~Hz}$.

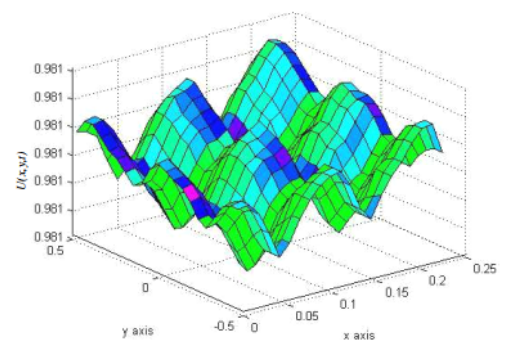

Figure 11. Mass Ratio $\left(\epsilon_{0}\right)=0.00$

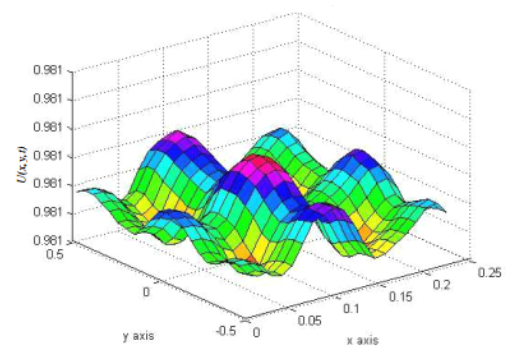

Figure 12. Mass Ratio $\left(\epsilon_{0}\right)=0.01$ 


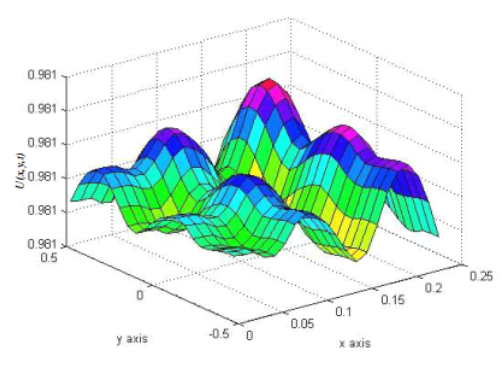

Figure 13. Mass $\operatorname{Ratio}\left(\epsilon_{0}\right)=0.025$

\section{Conclusion}

The effects of shear deformation and rotary inertia on the dynamics of moving concentrated loads on a rectangular plate with varying flexural rigidity and varying mass per unit area is considered in this study. The plate is resting on a Vlasov foundation. The fourth order partial differential equations which describes the system is reduced to a system of coupled fourth order ordinary differential equations using a method on the separation of variables. A approximate analytic solution to the problem for moving force and moving mass is obtained using a modification of the Struble's technique and then Laplace transform method. Results obtained from the study indicate that shear deformation, rotary inertia and mass per unit area had significant effect on the dynamics of the anisotropic plate transverse by moving load. The results also indicate that the amplitude of vibrations of the plate under moving mass is greater than that of the moving force for same values of shear modulus and rotary inertia. Results obtained in this study is consistent with results obtained by $[6,14-16]$.

Acknowledgments: The authors wish to express their profound gratitude to the reviewers for their useful comments on the manuscript.

Author Contributions: All authors contributed equally to the writing of this paper. All authors read and approved the final manuscript.

Conflicts of Interest: "The authors declare no conflict of interest."

\section{References}

[1] Sathyamoorthy, M \& Chia, C. Y. (1980). Effects of transverse shear and rotary inertia on large amplitude vibration of anisotropic skew plates. Journal of applied Mechanics, 47(1), 128-132.

[2] Koo, K. (2014). Effects of shear deformation and rotary inertia on the natural frequencies of axially loaded beams. Journal of Mechanical Science and Technology, 28(3), 849-857.

[3] Toorani, M. H. \& Lakis, A. A. (2000). General equations of anisotropic plates and shells including transverse shear deformations, rotary inertia and initial curvature effects. Journal of Sound and vibration, 237(4), 561-615.

[4] Mindlin, R.D., (1951). Influence of rotary inertia and shear on flexural motions of isotropic elastic plates. Journal of Applied Mechanics, 18(1), 31-38.

[5] Aiyesimi, Y.M, (2000). The analytical investigation of the effect of amplitude ratio on the dynamic response of a viscoelastic plate subject to a cyclic moving load. Journal of Science, Technology E Mathematics Education,3(2),79-84.

[6] Awodola, T. O. \& Omolofe, B. (2014). Response to concentrated moving masses of elastically supported rectangular plates resting on Winkler elastic foundation. Journal of Theoretical and Applied Mechanics, 44(3), 65-90.

[7] Esen, I. (2015). A new FEM procedure for transverse and longitudinal vibration analysis of thin rectangular plates subjected to a variable velocity moving load along an arbitrary trajectory.Latin American Journal of Solids and Structures 12, 808-830.

[8] Kocaturk, T. (1995). Rectangular anisotropic (orthotropic) plates on a tensionless elastic foundation. Mechanics of Composite Materials, 31(3), 378-386.

[9] Ozgan, K. (2018). Modelling Laminated Orthotropic Plate-Foundation Interaction Subjected To Moving Load Using Vlasov Model. Teknik Dergi/Technical Journal of Turkish Chamber of Civil Engineers, 29(2), 8317-8338.

[10] Vlasov, V. \& Leont'ev, N. (1966). Beams, plates, and shells on elastic foundations. Translated from Russian, Israel Program for Scientific Translations, Jerusalem.

[11] Ozgan, K. \& Dalogu, A. T. (2014). Dynamic response of thick plates on two parameter elastic foundation under time variable loading. International Journal of Engineering \& Applied Sciences (IJEAS), 6(4), 40-51

[12] Shadnam, M. R., Mofid, M., Akin, J. E. (2001). On the dynamic response of rectangular plate, with moving mass. Thin-Walled Structures 39 (2001), 797-806 
[13] Oni, S.T. \& Jimoh, A. (2016). Dynamic response to moving concentrated loads of non-uniform simply supported prestressed Bernoulli-Euler beam resting on bi-parametric subgrades. International Journal of Scientific E Engineering Research, 7(3), 754-770

[14] Awodola, T. O. \& Omolofe, B. (2018). Flexural motion of elastically supported rectangular plates under concentrated moving masses and resting on bi-parametric elastic foundation. Journal of Vibration Engineering E Technologies, 6(3), 165-177.

[15] Oni, S.T \& Awodola, T. O. (2011). Dynamic behaviour under moving concentrated masses of simply supported rectangular plates resting on variable Winkler elastic foundation. Latin American Journal of Solids $\mathcal{E}$ Structures, 8(1), 373-392.

[16] Omolofe, B. \& Oni, S. T. (2015). Transverse motions of rectangular plates resting on elastic foundation and under concentrated masses moving at varying velocities. Latin American Journal of Solids and Structures, 12, 1296-1318.

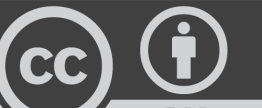

BY

(C) 2019 by the authors; licensee PSRP, Lahore, Pakistan. This article is an open access article distributed under the terms and conditions of the Creative Commons Attribution (CC-BY) license (http://creativecommons.org/licenses/by/4.0/). 